\title{
Deep Brain Stimulation for Substance Abuse
}

\author{
Nora Vanegas $•$ Kareem A. Zaghloul
}

Published online: 7 March 2015

(C) Springer International Publishing AG (outside the USA) 2015

\begin{abstract}
Substance abuse represents a significant public health issue, yet current standard treatments are largely ineffective. Deep brain stimulation (DBS) surgery may offer an effective interventional approach to treat substance abuse by enabling direct manipulation of neural circuits. DBS has been successfully used in patients with movement disorders and, more recently, in select psychiatric conditions. The ability of DBS surgery to address addictive behavior, however, critically relies upon fundamental knowledge of reward pathways within the brain. Recent evidence has provided insight into those pathways, and studies using DBS surgery in animal models of addiction and reports describing the successful use of DBS in humans with substance abuse disorders provide encouraging support for the feasibility of DBS in treating addiction. Here, we review the pathophysiological mechanisms of addiction and the currently available literature on the application of DBS for substance abuse.
\end{abstract}

Keywords Addiction - Substance abuse - Brain stimulation · Nucleus accumbens $\cdot$ Dopamine

This article is part of the Topical Collection on Neuromodulation

N. Vanegas

Office of the Clinical Director, Human Motor Control Section, National Institute of Neurological Disorders and Stroke, National Institutes of Health, Building 10, Room 7D42, 10 Center Drive, Bethesda, MD 20892-1414, USA

e-mail: nora.vanegas-arroyave@nih.gov

\section{K. A. Zaghloul $(\triangle)$}

Surgical Neurology Branch, National Institute of Neurological Disorders and Stroke, National Institutes of Health, Building 10, Room 3D20, 10 Center Drive, Bethesda, MD 20892-1414, USA e-mail: kareem.zaghloul@nih.gov

\section{N. Vanegas}

Human Motor Control Section, National Institute of Neurological Disorders and Stroke, National Institutes of Health,

Bethesda, MD 20892, USA

\section{Introduction}

Substance abuse is defined by the DSM-V as a cluster of cognitive, behavioral, and physiological symptoms indicating that an individual continues to use a substance despite significant substance-related problems. The diagnosis of substance abuse can be applied to ten substance classes, including alcohol, cannabis, hallucinogens, tobacco, opioids, sedatives, and hypnotics [1]. The adverse effects of illicit drug use include the acute effects of intoxication and overdose, and the development of dependence. Adverse long-term health effects of chronic drug use include chronic heart and liver disease, blood-borne bacterial and viral infections, and psychiatric disorders [2].

Accurate estimates of the prevalence of illicit drug use are challenging, particularly because of issues related to patient confidentiality and the potential legal repercussions. The 2011 world drug report by the United Nations Office on Drugs and Crime (UNODC) provides a wide range of prevalence estimates across countries and regions [2]. According to UNODC estimates, 149-271 million people aged 15-64 years (3.3 $6.1 \%$ ) had used an illicit drug at least once in 2009 [3]. Drug use varies across regions and, frequently, drug use involves more than one type of drug [3]. Alcohol and tobacco are the most frequently used drugs of abuse. Alcohol abuse has a prevalence of $4.65 \%$ in the USA [4], and worldwide, alcohol causes about $20-30 \%$ of esophageal cancer, liver cancer, liver cirrhosis, homicides, seizures, and motor vehicle accidents. During 2001-2005, excessive alcohol use accounted for approximately 80,000 deaths and 2.3 million years of potential life lost in the USA each year [5]. Overall, it has been estimated that alcohol abuse causes 1.8 million deaths each year and a loss of 58.3 million of disability-adjusted life years [6]. Similarly, cigarette smoking is the leading cause of preventable diseases and death in the USA, resulting in approximately 443,000 deaths and $\$ 193$ billion of direct health-care expenditures and productivity losses each year [7]. 
While less commonly used than either alcohol or tobacco, illicit drugs also represent a significant public health issue. Cannabis is likely the most widespread and commonly used illicit drug, and the estimated number of users around the world is between 125 and 203 million people (2.8-4.5\% of the global population aged 15-64) [2]. Estimates for the number of users of cocaine or opioids range from 14 to 21 million $(0.3-0.5 \%$ of the population aged 15-64 years) and 12-21 million people, respectively [2]. Besides the obvious issues related to drug dependence, health risks associated with heroin, commonly used by intravenous injections, are substantial and include an increased incidence of human immunodeficiency virus and hepatitis transmission [2]. Furthermore, the incidence of mental disorders, road-traffic accidents, suicides, and violence is increased in opioid, cocaine, and amphetamine users [8].

Traditional treatments for substance abuse include outpatient drug-free programs, therapeutic communities, chemical dependency programs, and cognitive behavioral therapy, in addition to pharmacological interventions such as methadone, naltrexone, and buprenorphine maintenance [8]. Despite these significant efforts and the availability of treatment options, treatments have been largely unsuccessful with abstinence rates at 1 year as low as 30-50 \% [9]. The reasons behind such high relapse rates are largely related to poor compliance, likely secondary to the association of substance abuse with social, physical, and psychiatric issues that influence the candidacy, adherence, and response to treatment. Given the significant burden that substance abuse imposes both on the individual and on the broader health-care system, there is a distinct need for more effective treatment options.

Interventional treatments that offer direct manipulation of the neural circuits implicated in substance abuse behavior may provide a reasonable alternative treatment approach. If successful, such approaches would have a significant impact on the individual, and more broadly could potentially lead to economic benefits, reduced crime rates, enhanced productivity, and lower rates of health-care utilization. One possible intervention is deep brain stimulation, which allows direct modulation of neural circuits and has been successfully deployed in addressing symptoms for patients with movement disorders. Deep brain stimulation (DBS) has recently been used for other pathophysiologically related psychiatric conditions, suggesting its possible effectiveness for the treatment of substance abuse. Based on the pathophysiological mechanisms of addiction, several targets have therefore been proposed for the treatment of substance abuse using DBS. Here, we review the current available literature on the application of DBS for the treatment of substance abuse.

\section{Mechanisms of Addiction}

Most addictive substances either trigger pleasure or relieve distress [10•], effects that play a pivotal role in driving the desire for repeated use. While initial experimentation with drugs of abuse is largely a voluntary behavior, continued drug use gradually impairs neural function, and eventually impacts the capacity to exert free will and turns drug use into a compulsive behavior [11•]. Furthermore, repeated and frequent drug consumption leads to the development of tolerance, which decreases the positive reinforcing effects [12••] of a substance and leads to increases in dosage [13] (Table 1). Similarly, negative reinforcement, related to the experience of withdrawal symptoms, plays an important role in the development of substance abuse, as an individual continues to use a substance in order to avoid the consequences of drug withdrawal $[13,14]$. The addiction phenotype (compulsive drug consumption, impaired self-control, and behavioral inflexibility) therefore represents an unbalanced interaction between brain regions implicated in goal-directed behaviors. This complex network includes such structures as the striatum, ventral tegmental area (VTA), substantia nigra (SN), amygdala, hippocampus, prefrontal cortex (PFC), and the lateral habenula (LHb) (Fig. 1). And central to the modulation of reward [15], emotional reactivity, and executive function within this network is dopamine (DA).

Dopaminergic projections from the VTA and SN to the striatum serve to reinforce responses to both primary rewards

Table 1 Definitions of addiction-related terminology

\begin{tabular}{|c|c|}
\hline Term & Definition \\
\hline Extinction & $\begin{array}{l}\text { Extinction is an important process of learning } \\
\text { that is typically defined as the removal of } \\
\text { reinforcers and/or biologically relevant } \\
\text { stimuli from a previously established } \\
\text { stimulus relationship that results in the } \\
\text { reduction in responding [44] }\end{array}$ \\
\hline Negative reinforcement & $\begin{array}{l}\text { The process by which removal of an aversive } \\
\text { stimulus (e.g., emotional state of drug } \\
\text { withdrawal) increases the probability of a } \\
\text { response (e.g., dependence) [12••] }\end{array}$ \\
\hline Positive reinforcement & $\begin{array}{l}\text { The process by which presentation of a } \\
\text { stimulus, usually pleasant, increases the } \\
\text { probability of a response }[12 \cdot \bullet]\end{array}$ \\
\hline Reinstatement & $\begin{array}{l}\text { Reinstatement refers to the reappearance } \\
\text { of the conditioned response following } \\
\text { post-extinction exposure to the } \\
\text { unconditional stimuli [44] }\end{array}$ \\
\hline Reinstatement & $\begin{array}{l}\text { Reinstatement refers to the reappearance } \\
\text { of the conditioned response following } \\
\text { post-extinction exposure to the } \\
\text { unconditional stimuli [44] }\end{array}$ \\
\hline Reward & $\begin{array}{l}\text { Induced state that subsequently leads to } \\
\text { a conditioned approach behavior [15] }\end{array}$ \\
\hline Withdrawal & $\begin{array}{l}\text { Development of a substance-specific } \\
\text { maladaptive behavioral change, with } \\
\text { physiological and cognitive symptoms, } \\
\text { that is due to the cessation of, or reduction } \\
\text { in, heavy and prolonged substance use }[14\end{array}$ \\
\hline
\end{tabular}


Fig. 1 Brain structures involved in reward circuits implicated in substance abuse disorders. Red arrows represent dopaminergic pathways from the VTA and $S N$

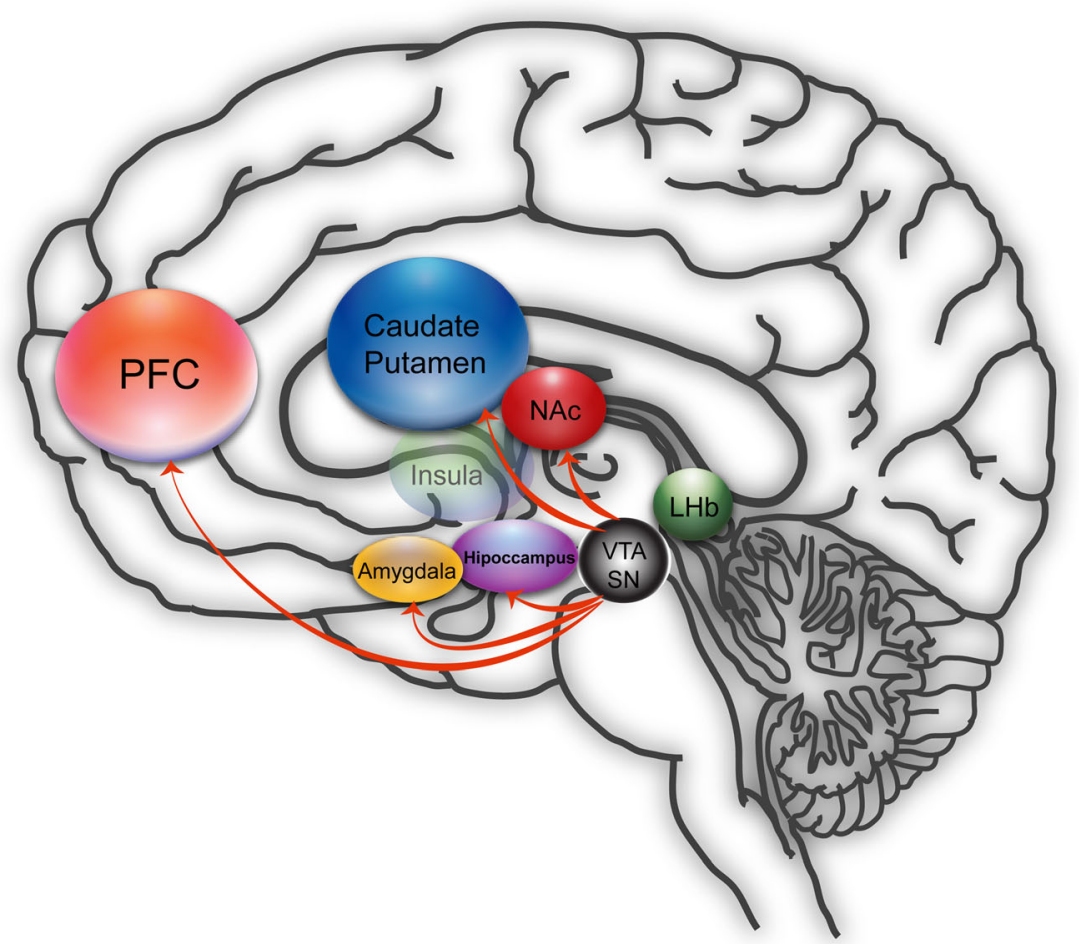

(e.g., food) and secondary rewards (e.g., money), and are fundamental for learning from past experiences and coordinating behavioral responses [16]. Either directly or indirectly, all addictive drugs have the power to cause large and transient increases in DA from VTA neurons that project primarily to the nucleus accumbens (NAc) of the ventral striatum, but also to the dorsal striatum, amygdala, hippocampus, hypothalamus, lateral septal area, and PFC [10•]. Human positron emission tomography studies have demonstrated that nicotine, alcohol, and cannabis can increase DA in the dorsal and ventral striatum, and that these increases are proportional to the intensity of the subjective experience of euphoria caused by the drug [10•]. However, the role of DA in reward is also related to its ability to encode the prediction of reward, the imprinting of incentive value to reinforcers, and the facilitation of learning of reward associations through its modulation of other subcortical and cortical brain regions [10•]. Drugs of abuse increase dopamine release in a more prolonged and unregulated way than natural stimuli [12••]. Furthermore, animal studies have demonstrated that a single in vivo exposure to cocaine induces long-term potentiation (LTP) of $\alpha$-amino3-hydroxy-5-methyl-4-isoxazolepropionic acid (AMPA)receptor-mediated currents at excitatory synapses onto dopamine cells in the VTA [17]. These alterations result in changes in synaptic plasticity within the dopaminergic system, which ultimately modify the normal learning mechanisms of the individual and result in a form of habit learning that persists even under significant adverse consequences $[12 \bullet \bullet$.
Several other circuits have been implicated in the neuroadaptive changes of the addiction cycle. The dorsal striatum may play a significant role in the cue- or context-dependent craving given its role in habit learning and action initiation $[12 \bullet \cdot]$. The right dorsolateral and inferior frontal cortices have demonstrated increased activation in cocaine users after an intravenous injection of cocaine, with a coincident improvement in a motor response inhibition task [18]. Hence, some of the acute effects of cocaine could be related to a transient reversal of the chronic hypofunction in the impulse control circuitry. The amygdala plays a pivotal role in both structural and functional adaptations that underlie drug-related memories [19]. And the anterior insula, reciprocally connected to several limbic regions including the ventromedial prefrontal cortex, amygdala, and ventral striatum, appears to integrate autonomic and visceral information with emotion and motivation, and therefore may be involved in motivational processes that promote addiction, such as conscious urges and the decision to relapse [20]. Indeed, insular reactivity has been suggested to serve as a biomarker to help predict substance abuse relapse [12••]. Finally, glutamatergic inputs from the LHb to the VTA have also been implicated in the pathophysiology of addiction [21]. Inhibition of dopaminergic VTA signaling by the LHb enables learning avoiding behaviors when an aversive stimulus or negative feedback is provided [10•]. The chronic use of addictive drugs leads to habenular hyperactivity, which may promote a negative emotional state during drug withdrawal [10•].

All together, the development of a change in the firing of mesolimbic dopamine neurons appears to begin with the first 
administration of the drug, develops into LTP first in the VTA then NAc, and via feedback loops, subsequently engages the dorsal striatum. Long-term changes in the amygdala and medial PFC may follow, and combined with chronic dysregulation of higher-order cognitive processes, may facilitate the ability of stress to serve as a powerful drive for drug-seeking behavior, even months after drug withdrawal [12••]. Hence, interventions targeted to disrupt this cycle may be necessary to effectively manipulate and address addictive behaviors. Direct electrical stimulation of structures within this network may offer one possible therapeutic approach for modulating the aberrant neural circuits triggered by substance abuse. Given that the NAc receives and transmits information between a variety of systems that promote distinct patterns of behavior related to motivation and goal seeking, this structure may be an important candidate target for modulatory interventions [22].

\section{DBS for Psychiatric Disorders}

DBS surgery has gained the most traction as a first-line treatment for patients with movement disorders. The first systematic application of high-frequency DBS for the treatment of Parkinsonian and essential tremor dates back to 1987 and involved targeted stimulation of the thalamic ventral intermediate (Vim) nucleus [23]. Since that initial study, other targets, including the subthalamic nucleus (STN) and the globus pallidus pars interna (GPi), have emerged as basal ganglia targets whose stimulation has proven to benefit other Parkinsonian symptoms including rigidity and bradykinesia. DBS for the treatment of Parkinson's disease (PD) has shown to be superior to the best medical treatment in the control of motor fluctuations and dyskinesia, and in the improvement of quality of life [24-26]. DBS therapy of the GPi has also shown significant and sustained success in the treatment of primary and cervical dystonia, and emerging evidence suggests a benefit in certain types of secondary dystonia such as tardive dystonia, dyskinetic cerebral palsy, and neurodegeneration with brain iron accumulation (NBIA) [27].

Building upon these initial successes using DBS, investigators have more recently trialed DBS for psychiatric disorders. In part, this was prompted by initial reports demonstrating the improvement in obsessive-compulsive disorder (OCD) symptoms in patients with PD treated with STN DBS [28, 29]. Today, DBS has been shown to be effective in the treatment of both Tourette's syndrome [30] and OCD [31••]. In one study of 15 patients who underwent DBS of the ventral striatum/ ventral capsule, depressive symptoms were significantly improved [32]. Although follow-up durations varied, at least five patients met criteria for remission and eight met criteria for clinical response [32]. Similar results from an open label trial of DBS of the subcallosal cingulate were recently reported.
After 2 years of chronic stimulation, a group of 17 patients with unipolar or bipolar depression exhibited high response and remission rates (92 and $58 \%$, respectively), with no significant relapse in those patients achieving remission [33]. Notably, in a follow-up study, diffusion tensor imaging (DTI) was used to identify white matter tracts traveling through the activation volumes in 16 of the original 17 patients. All responders had activation volumes that involved three specific white matter pathways [34]. These data importantly suggest a target selection strategy based on network connectivity and raise the possibility that optimal electrode implantation and stimulation parameter selection for DBS in psychiatric disease may critically rely on the accurate identification of relevant white matter tracts.

\section{Animal Studies of DBS for Substance Abuse}

Several animal studies have reported the effects of brain stimulation on drug-seeking behaviors. Based on the known reward and addiction pathways, most studies have focused on stimulation of the NAc, although low- and high-frequency stimulation of the medial $\mathrm{PFC}$ in rats also demonstrates reductions in the motivation for cocaine [35]. In rodents, the NAc has two morphologically and functionally distinct subunits, a shell and a core [36]. Stimulation of either the NAc shell or core region in the normal brain of rodents can elicit different effects on drug-seeking behavior. In primates and humans, the shell area has regressed and is no longer clearly distinguishable or functionally distinct [36].

In one study, chronic bilateral DBS of the NAc core significantly attenuated heroin-seeking behavior in rats, and unilateral DBS of the right NAc core provided almost equivalent effects [37]. Importantly, stimulation of the NAc had no significant effect on motor activity and spatial learning. Similar results were observed in a separate study examining morphine use in rats 7 to 10 days after high-frequency NAc DBS [38]. DBS of the NAc shell attenuates the reinstatement of drugseeking behavior on rats re-exposed to cocaine [39] and morphine [40]. DBS of the NAc accelerated the innate decay of drug craving in morphine-dependent rats without, again, significantly influencing learning and memory as assessed using the Morris water maze task [40]. And DBS of the NAc reduced alcohol consumption in $\mathrm{P}$ rats that spontaneously consume alcohol without requiring reinforcement, both acutely and after a period of alcohol deprivation [41, 42]. More recently, DBS of the NAc shell was directly compared to pharmacological inactivation through bilateral infusions of a GABA agonist in $\mathrm{P}$ rats [43]. Alcohol consumption decreased by $55 \%$ in the bilateral GABA-infusion group and by $47 \%$ in those receiving DBS of the NAc shell [43].

Abnormalities of the LHb have been demonstrated in several psychiatric disorders such as depression, psychosis, and 
drug addiction. Over-activation of the LHb following continuous subcutaneous cocaine exposure leads to axonal degeneration of the fasciculus retroflexus (FR), which connects the NA to the VTA [21]. DBS of the LHb significantly attenuates cocaine-seeking behavior in rats, suggesting that stimulation of the LHb interferes with reward processes mediated through these interactions and subsequent drug-induced plasticity of the VTA [21]. Furthermore, DBS of the LHb during maintenance, extinction and reinstatement [44] reduced cocaineseeking behavior at the lower and intermediate, but not at the highest cocaine doses [21]. Hence, the efficacy of LHb DBS may depend on the severity of the addiction, and therefore on the resulting FR degeneration [45].

Animal studies therefore suggest that stimulation of the $\mathrm{NAc}, \mathrm{LHb}$, and PFC may successfully decrease drugseeking behaviors in animals without resulting in significant side effects. While encouraging, the possible contributors and consequences of addictive behaviors in humans are likely more complicated than in animals. Analogous stimulation paradigms should therefore account for technical and dimensional differences that could have important implications when translated to humans.

\section{Human Studies}

Stereotactic surgery was first used as a treatment for substance abuse as early as 1999, and involved creating a lesion in the cingulate gyrus. In 335 patients, the procedure resulted in a $60 \%$ rate of total remission [46]. Stereotactic ablation of the NAc similarly demonstrated significant results in the treatment of opioid abuse in China between 2000 and 2004 [37]. No severe complications were reported, and the relapse rate varied from $15.4 \%$ to $54.8 \%$ after a mean follow-up of 6 to 12 months. The procedure was subsequently prohibited due to the lack of appropriate experimental models and long-term follow-up studies [47]. However, the long-term outcomes of those patients $(n=272)$ were subsequently reported, and 1year relapse rates were only $30.5 \%$ [48]. In the crosssectional follow-up that included 100 of the initial 272 patients, the 5-year relapse rate was $42 \%$ [48].

While these studies each had their particular design flaws, they nevertheless suggested that ablative procedures may be of potential benefit in the treatment of substance abuse [49]. Indeed, patients with damage to the insula, an area associated with motivational processes that drive addictive behavior, exhibited significantly higher rates of smoking cessation than patients with non-insular lesions [50]. This is consistent with other reports associating insular lesions with smoking cessation [51, 52]. Ablative procedures, however, are irreversible, and lesions arising from vascular events are arbitrary and uncontrolled. Given the efficacy of DBS for movement disorders and select psychiatric disorders, neuromodulation rather than ablation may offer a more attractive interventional alternative. Indeed, some patients with PD treated with STN DBS exhibited improvements in symptoms related to dopamine dysregulation syndrome [53, 54], a form of addiction involving dopamine treatment $[55 \cdot \bullet]$. Several studies have therefore retrospectively evaluated the effects of DBS on patients with psychiatric disorders and comorbid substance abuse, including alcohol and nicotine.

Patients treated with DBS of the NAc for mood disorders exhibited a higher rate of smoking cessation, compared to unaided smoking cessation rates in the general population [56*0]. In a report evaluating NAc DBS in a patient with an anxiety disorder and alcohol abuse, the patient experienced remarkable improvement of his alcohol dependency at 12 months of follow-up, with only occasional consumption of alcohol [57]. Similarly, a 47-year-old woman with history of severe refractory OCD and morbid obesity underwent NA DBS therapy. In addition to improvements in her psychiatric symptoms, she was able to quit smoking after 10 months of therapy and started a weight loss program, reaching her goal weight and body mass index after losing $44 \mathrm{~kg}$ [58].

While the aforementioned studies have examined the effects of DBS on substance abuse in patients receiving DBS for comorbid psychiatric conditions, to date, no sham-controlled studies have explicitly evaluated the long-term effects of DBS on patients with substance abuse as a primary outcome. Several case reports, however, suggest that such a study, if conducted, may prove successful. In a report of a 24-year-old man with history of heroin dependence receiving NAc DBS, regular heroin use that had been refractory to standard treatment subsided after surgical intervention and stimulation therapy [59]. The device was subsequently turned off and the pulse generator removed after 3 years, yet the patient continued to abstain from heroin after 3.5 years of follow-up. In a separate report of a 47-year-old man with treatment-refractory heroin dependence for 22 years, bilateral NAc DBS significantly reduced drug use and craving [60]. And in a third case report, a 69-year-old male with a 30-year history of refractory alcohol dependence underwent DBS surgery of the NAc, resulting in complete cessation of alcohol consumption 1 year after the start of stimulation therapy [61]. Notably, the patient also exhibited improvements in error-related negativity, an electroencephalographic signal associated with the commission of errors, indicating an improvement of the functional integrity of the performance-monitoring system [61].

In the largest case report series, three patients with refractory alcohol dependence received bilateral NAc DBS [62]. All patients experienced a significant reduction in alcohol craving without significant adverse effects. Two patients remained abstinent at 1-year follow-up, and the alcohol consumption was reduced considerably in the third patient [62]. In a follow-up study, these three patients were included in a case series of five patients with severe refractory alcohol addiction who received 
bilateral DBS of the NAc [13]. All five patients had a history of alcoholism for more than 10 years and had unsuccessfully attempted therapy with acamprosate, naltrexone, or disulfiram. Remarkably, all exhibited complete remission of alcohol cravings with stimulation with no adverse events or permanent side effects. Two of the patients had remained completely abstinent for more than 4 years, while one patient had a transient episode of hypomania that resolved after readjusting his programming settings [13].

In summary, animal studies have shown promising results for the treatment of drug-seeking behaviors using DBS of several targets known to play an important role in the reward circuit, and individual human case reports consistently suggest that this intervention may offer a promising alternative to standard therapy. Yet despite these encouraging signs, there still remain no reported randomized sham-controlled studies evaluating the effects of DBS for substance abuse in humans. Animal studies and these individual case reports suggest that the NAc may represent the first candidate structure for such a trial investigating DBS therapy in the treatment of substance abuse. DBS of the NAc has already demonstrated efficacy in the treatment of anxiety disorders, principally OCD, and the pathophysiologic similarities between substance abuse and other psychiatric disorders of reward circuitry raise the intriguing possibility that stimulation of this target may be effective for addiction disorders as well. It should be noted, however, that disorders of substance abuse likely involve a complex interaction of structures within the reward circuit, and so future studies should also explore the efficacy of DBS on other targets and white matter tracts within this network.

\section{Mechanisms of DBS for Substance Abuse}

DBS therapy for movement disorders has been standard care for over a decade, yet the precise mechanisms responsible for the therapeutic effects of DBS on neurodegenerative and psychiatric conditions remain unclear. Hence, how exactly DBS would address disorders of addiction is unknown. Given the postulated pathophysiology of addictive behaviors, one possibility is that DBS may normalize disrupted VTA or NAc activity, but studies investigating this in detail are limited.

Electrophysiological animal studies suggest a reduced firing in orbitofrontal cortex pyramidal cells and enhanced synchronicity of the thalamo-cortical circuit after high-frequency NAc DBS [9]. NAc DBS may therefore modulate the activity of specific pathways within a dysfunctional neuronal network [9]. And in a study investigating changes in AMRI BOLD activation following NAc DBS in 13 patients with OCD, NAc activity that had been reduced in OCD patients with stimulation off returned to levels observed in age-matched controls following stimulation [63]. Resting state fMRI in these patients demonstrated that DBS reduced connectivity between the NAc and the prefrontal cortex, and DBS also attenuated increases in low-frequency oscillations over the frontal cortex provoked by behavioral stimuli in a reward anticipation task [63]. One possibility, then, is that DBS reduces abnormal NAc activity and connectivity, as well as the frontal brain response triggered by symptom-provoking events.

Furthermore, studies of addicted individuals have also shown decreased activity in the cingulate gyrus and the dorsolateral prefrontal cortex, changes that presumably affect the process of inhibitory control [9]. Modulating neuronal activity within this network could therefore lead to an increase in selfcontrol. These mechanisms are not mutually exclusive, and both could contribute to the reported effects. Moreover, different brain regions and different classes of cells may be affected differently by high frequency stimulation, although in what manner is largely unknown [9]. Future research on the mechanisms of addiction and the possible effects of DBS based on newly developed strategies such as network connectivity [34] could guide the search of an optimal target for both substance abuse and other psychiatric conditions.

\section{Conclusion}

The central mechanism of addiction is based on the dysfunction of the reward circuit, similar to the disruptions observed in other related psychiatric conditions. The evidence that addictive behaviors may improve after the use of DBS for other psychiatric and movement disorder conditions suggests its applicability for the treatment of addiction. Animal studies have mostly focused on several targets known to play an important role in reward networks. These studies have been complemented by several human case reports that have consistently demonstrated a reduction in substance abuse without significant side effects after DBS of the NAc. Further advances in understanding the mechanisms responsible for the development of substance abuse and in the possible mechanisms underlying high-frequency stimulation in these disorders will be critical for establishing this therapy as an effective treatment option for substance abuse and in guiding the discovery of other possible targets of neuromodulation. The preliminary evidence regarding the safety and efficacy of DBS for the treatment of substance abuse disorders, however, is both promising and exciting.

\section{Compliance with Ethics Guidelines}

Conflict of Interest Kareem Zaghloul and Nora Vanegas have no relevant disclosures.

Human and Animal Rights and Informed Consent This article does not contain any studies with human or animal subjects performed by any of the authors. 


\section{References}

Papers of particular interest, published recently, have been highlighted as:

- Of importance

- Of major importance

1. American Psychiatric Association \& American Psychiatric Association. DSM-5 Task Force. Diagnostic and statistical manual of mental disorders: DSM-5. 5th ed. American Psychiatric Association. 2013.

2. Degenhardt L, Hall W. Extent of illicit drug use and dependence, and their contribution to the global burden of disease. Lancet. 2012;379:55-70. doi:10.1016/S0140-6736(11)61138-0.

3. UNODC. United Nations Office of Drug and Crime. World drug report. 2011.

4. WHO. National epidemiologic survey on alcohol and related conditions (NESARC). 2006.

5. Bouchery EE, Harwood HJ, Sacks JJ, Simon CJ, Brewer RD. Economic costs of excessive alcohol consumption in the U.S., 2006. Am J Prev Med. 2011;41:516-24. doi:10.1016/j.amepre. 2011.06.045.

6. Room R. Alcohol in developing societies: a public health approach. World Health Organization 2002.

7. Frieden TR, Centers for Disease, C. \& Prevention. CDC health disparities and inequalities report-United States, 2013. Foreword. Morb Mortal Wkly Rep Surveill Summ. 2013;62 Suppl 3:1-2.

8. In pathways of addiction: opportunities in drug abuse research. The National Academies Collection: Reports funded by National Institutes of Health 1996.

9. Luigjes $\mathbf{J}$ et al. Deep brain stimulation in addiction: a review of potential brain targets. Mol Psychiatry. 2012;17:572-83. doi:10. 1038/mp.2011.114.

10. Volkow ND, Wang GJ, Tomasi D, Baler RD. Unbalanced neuronal circuits in addiction. Curr Opin Neurobiol. 2013;23:639-48. doi:10. 1016/j.conb.2013.01.002. Describes the current understanding of the circuitry underlying addiction.

11. Volkow ND, Baler RD. Addiction science: uncovering neurobiological complexity. Neuropharmacology. 2014;76(Pt B):235-49. doi:10.1016/j.neuropharm.2013.05.007. Highlights some of the most significant and recent findings in addiction research.

12.• Koob GF, Volkow ND. Neurocircuitry of addiction. Neuropsychopharmacology. 2010;35:217-38. doi:10.1038/npp. 2009.110. Largest case series of successful DBS treatment for severe alcoholism.

13. Voges J, Muller U, Bogerts B, Munte T, Heinze HJ. Deep brain stimulation surgery for alcohol addiction. World Neurosurg. 2013;80(S28):e21-31. doi:10.1016/j.wneu.2012.07.011.

14. Miller NS, Summers GL, Gold MS. Cocaine dependence: alcohol and other drug dependence and withdrawal characteristics. J Addict Dis. 1993;12:25-35. doi:10.1300/J069v12n01 03.

15. Ikemoto S, Bonci A. Neurocircuitry of drug reward. Neuropharmacology. 2014;76(Pt B):329-41. doi:10.1016/j. neuropharm.2013.04.031.

16. Dichter GS, Damiano CA, Allen JA. Reward circuitry dysfunction in psychiatric and neurodevelopmental disorders and genetic syndromes: animal models and clinical findings. J Neurodev Disord. 2012;4:19. doi:10.1186/1866-1955-4-19.

17. Ungless MA, Whistler JL, Malenka RC, Bonci A. Single cocaine exposure in vivo induces long-term potentiation in dopamine neurons. Nature. 2001;411:583-7. doi:10.1038/35079077.
18. Garavan H, Kaufman JN, Hester R. Acute effects of cocaine on the neurobiology of cognitive control. Philos Trans R Soc Lond B Biol Sci. 2008;363:3267-76. doi:10.1098/rstb.2008.0106.

19. Luo YX, Xue YX, Shen HW, Lu L. Role of amygdala in drug memory. Neurobiol Learn Mem. 2013;105:159-73. doi:10.1016/j. nlm.2013.06.017.

20. Naqvi NH, Bechara A. The hidden island of addiction: the insula. Trends Neurosci. 2009;32:56-67. doi:10.1016/j.tins.2008.09.009.

21. Friedman A et al. Electrical stimulation of the lateral habenula produces enduring inhibitory effect on cocaine seeking behavior. Neuropharmacology. 2010;59:452-9. doi:10.1016/j.neuropharm. 2010.06.008.

22. Floresco SB. The nucleus accumbens: an interface between cognition, emotion, and action. Annu Rev Psychol. 2014. doi:10.1146/ annurev-psych-010213-115159.

23. Benabid AL et al. Long-term suppression of tremor by chronic stimulation of the ventral intermediate thalamic nucleus. Lancet. 1991;337:403-6.

24. Castrioto A, Moro E. New targets for deep brain stimulation treatment of Parkinson's disease. Expert Rev Neurother. 2013;13:131928. doi:10.1586/14737175.2013.859987.

25. Deuschl $\mathrm{G}$ et al. A randomized trial of deep-brain stimulation for Parkinson's disease. N Engl J Med. 2006;355:896-908. doi:10. 1056/NEJMoa060281.

26. Weaver FM et al. Bilateral deep brain stimulation vs best medical therapy for patients with advanced Parkinson disease: a randomized controlled trial. Jama. 2009;301:63-73. doi:10.1001/jama.2008. 929.

27. Mills KA, Starr PA, Ostrem JL. Neuromodulation for dystonia: target and patient selection. Neurosurg Clin N Am. 2014;25:59 75. doi:10.1016/j.nec.2013.08.014.

28. Mallet L et al. Compulsions, Parkinson's disease, and stimulation. Lancet. 2002;360:1302-4. doi:10.1016/S0140-6736(02)11339-0.

29. Fontaine D et al. Effect of subthalamic nucleus stimulation on obsessive-compulsive disorder in a patient with Parkinson disease. Case Rep J Neurosurg. 2004;100:1084-6. doi:10.3171/jns.2004. 100.6.1084.

30. Kim W, Pouratian N. Deep brain stimulation for Tourette syndrome. Neurosurg Clin N Am. 2014;25:117-35. doi:10.1016/j.nec.2013. 08.009 .

31.• Fitzgerald $\mathrm{PB}$. The emerging use of brain stimulation treatments for psychiatric disorders. Aust N Z J Psychiatry. 2011;45:923-38. doi: 10.3109/00048674.2011.615294. Describes a novel method for patient-specific target and stimulation parameter selection.

32. Malone Jr DA et al. Deep brain stimulation of the ventral capsule/ ventral striatum for treatment-resistant depression. Biol Psychiatry. 2009;65:267-75. doi:10.1016/j.biopsych.2008.08.029.

33. Holtzheimer PE et al. Subcallosal cingulate deep brain stimulation for treatment-resistant unipolar and bipolar depression. Arch Gen Psychiatry. 2012;69:150-8. doi:10.1001/archgenpsychiatry.2011. 1456.

34. Riva-Posse $\mathrm{P}$ et al. Defining critical white matter pathways mediating successful subcallosal cingulate deep brain stimulation for treatment-resistant depression. Biol Psychiatry. 2014. doi:10.1016/ j.biopsych.2014.03.029.

35. Levy D et al. Repeated electrical stimulation of reward-related brain regions affects cocaine but not "natural" reinforcement. J Neurosci: Off J Soc Neurosci. 2007;27:14179-89. doi:10.1523/JNEUROSCI. 4477-07.2007.

36. Sturm V et al. The nucleus accumbens: a target for deep brain stimulation in obsessive-compulsive- and anxiety-disorders. J Chem Neuroanat. 2003;26:293-9.

37. Guo L et al. DBS of nucleus accumbens on heroin seeking behaviors in self-administering rats. Drug Alcohol Depend. 2013;129: 70-81. doi:10.1016/j.drugalcdep.2012.09.012. 
38. Liu HY et al. Chronic deep brain stimulation in the rat nucleus accumbens and its effect on morphine reinforcement. Addict Biol. 2008;13:40-6. doi:10.1111/j.1369-1600.2007.00088.x.

39. Vassoler FM et al. Deep brain stimulation of the nucleus accumbens shell attenuates cocaine priming-induced reinstatement of drug seeking in rats. J Neurosci: Off J Soc Neurosci. 2008;28:8735-9. doi:10.1523/JNEUROSCI.5277-07.2008.

40. Ma Y, Chen N, Wang HM, Meng FG, Zhang JG. Inhibition of the reinstatement of morphine-induced place preference in rats by highfrequency stimulation of the bilateral nucleus accumbens. Chin Med J (Engl). 2013;126:1939-43.

41. Henderson MB et al. Deep brain stimulation of the nucleus accumbens reduces alcohol intake in alcohol-preferring rats. Neurosurg Focus. 2010;29:E12. doi:10.3171/2010.4.FOCUS10105.

42. Knapp CM, Tozier L, Pak A, Ciraulo DA, Kornetsky C. Deep brain stimulation of the nucleus accumbens reduces ethanol consumption in rats. Pharmacol Biochem Behav. 2009;92:474-9. doi:10.1016/j. pbb.2009.01.017.

43. Wilden JA et al. Reduced ethanol consumption by alcoholpreferring $(\mathrm{P})$ rats following pharmacological silencing and deep brain stimulation of the nucleus accumbens shell. J Neurosurg. 2014;120:997-1005. doi:10.3171/2013.12.JNS13205.

44. Widholm J. Extinction learning as a model of drug treatment and relapse: a behavioral overview. Open Addict J. 2010;3:57-62.

45. Lax E et al. Neurodegeneration of lateral habenula efferent fibers after intermittent cocaine administration: implications for deep brain stimulation. Neuropharmacology. 2013;75:246-54. doi:10. 1016/j.neuropharm.2013.06.034.

46. Orellana C. Controversy over brain surgery for heroin addiction in Russia. Lancet Neurol. 2002;1:333.

47. Qiu J. China clamps down on controversial therapies. Lancet. 2009;373:1834-5.

48. Li $\mathrm{N}$ et al. Nucleus accumbens surgery for addiction. World Neurosurg. 2013;80(S28):e29-19. doi:10.1016/j.wneu.2012.10. 007.

49. Pierce RC, Vassoler FM. Deep brain stimulation for the treatment of addiction: basic and clinical studies and potential mechanisms of action. Psychopharmacology. 2013;229:487-91. doi:10.1007/ s00213-013-3214-6.

50. Naqvi NH, Rudrauf D, Damasio H, Bechara A. Damage to the insula disrupts addiction to cigarette smoking. Science. 2007;315: 531-4. doi:10.1126/science.1135926.

51. Hefzy H, Silver RW, Silver B. The no smoking sign - insular infarction. J Neuroimaging. 2011;21:e169-70. doi:10.1111/j.15526569.2010.00486.x
52. Suner-Soler R et al. Smoking cessation 1 year poststroke and damage to the insular cortex. Stroke. 2012;43:131-6. doi:10.1161/ STROKEAHA.111.630004.

53. Witjas T et al. Addiction in Parkinson's disease: impact of subthalamic nucleus deep brain stimulation. Mov Disord: Off J Mov Disord Soc. 2005;20:1052-5. doi:10.1002/mds.20501.

54. Knobel D, Aybek S, Pollo C, Vingerhoets FJ, Berney A. Rapid resolution of dopamine dysregulation syndrome (DDS) after subthalamic DBS for Parkinson disease (PD): a case report. Cogn Behav Neurol: Off J Soc Behav Cogn Neurol. 2008;21:187-9. doi:10.1097/WNN.0b013e318185e6e2.

55.• Lawrence AD, Evans AH, Lees AJ. Compulsive use of dopamine replacement therapy in Parkinson's disease: reward systems gone awry? Lancet Neurol. 2003;2:595-604. Case report of successful treatment for heroin abuse after NAc DBS.

$56 . \bullet$ Kuhn J et al. Observations on unaided smoking cessation after deep brain stimulation of the nucleus accumbens. Eur Addict Res. 2009;15:196-201. doi:10.1159/000228930. Case report of successful treatment of heroin abuse with NAc DBS.

57. Kuhn J et al. Remission of alcohol dependency following deep brain stimulation of the nucleus accumbens: valuable therapeutic implications? J Neurol Neurosurg Psychiatry. 2007;78:1152-3. doi: 10.1136/jnnp.2006.113092.

58. Mantione M, van de Brink W, Schuurman PR, Denys D. Smoking cessation and weight loss after chronic deep brain stimulation of the nucleus accumbens: therapeutic and research implications: case report. Neurosurgery. 2010;66:E218. doi:10.1227/01.NEU. 0000360570.40339.64. discussion E218.

59. Zhou H, Xu J, Jiang J. Deep brain stimulation of nucleus accumbens on heroin-seeking behaviors: a case report. Biol Psychiatry. 2011;69:e41-2. doi:10.1016/j.biopsych.2011.02.012.

60. Valencia-Alfonso CE et al. Effective deep brain stimulation in heroin addiction: a case report with complementary intracranial electroencephalogram. Biol Psychiatry. 2012;71:e35-7. doi:10.1016/j. biopsych.2011.12.013.

61. Kuhn J et al. Successful deep brain stimulation of the nucleus accumbens in severe alcohol dependence is associated with changed performance monitoring. Addict Biol. 2011;16:620-3. doi:10.1111/ j.1369-1600.2011.00337.x.

62. Muller UJ et al. Successful treatment of chronic resistant alcoholism by deep brain stimulation of nucleus accumbens: first experience with three cases. Pharmacopsychiatry. 2009;42:288-91. doi:10. 1055/s-0029-1233489.

63. Figee $\mathrm{M}$ et al. Deep brain stimulation restores frontostriatal network activity in obsessive-compulsive disorder. Nat Neurosci. 2013;16: 386-7. doi:10.1038/nn.3344. 